LOCKHEED MATTIN

ENVIRONMENTAL

RESTORATION

PROGRAM

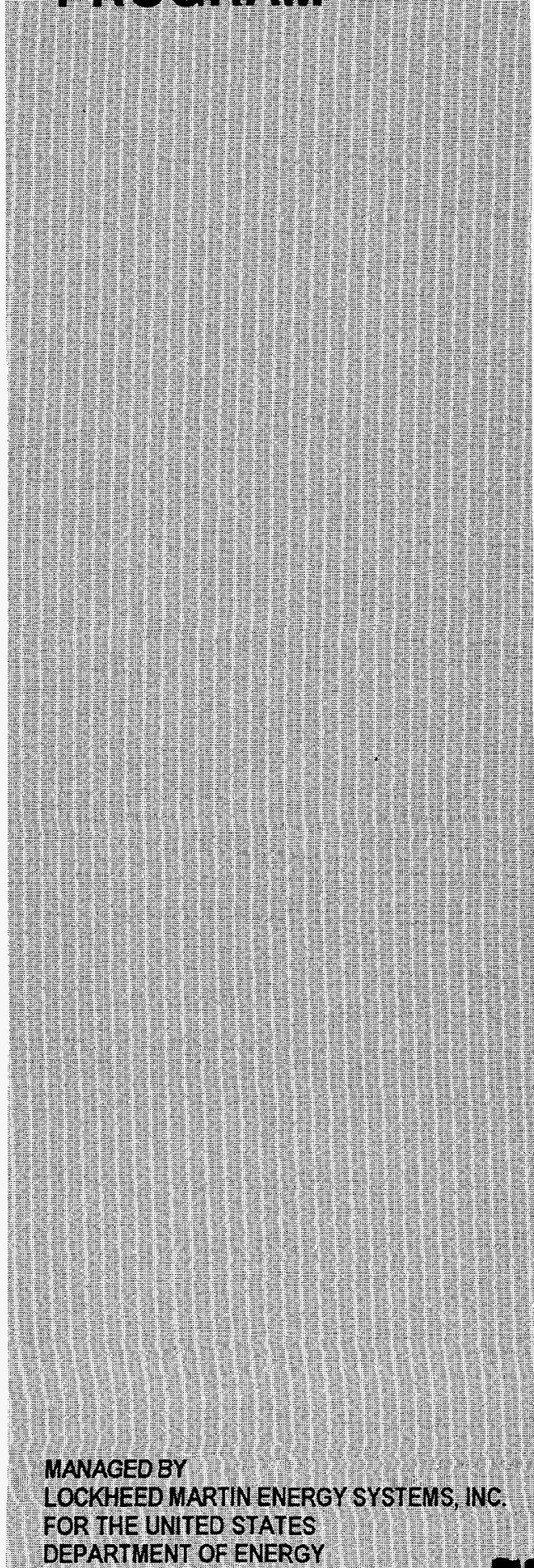

DEPARTMENT OF ENERGY
Y/ER-287

\section{Health and Safety Plan for Phase II of the Bear Creek Valley Treatability Study Oak Ridge Y-12 Plant, Oak Ridge, Tennessee}

\author{
RECEIVED \\ JUL $\cap 29997$ \\ OSTI
}

This document has been approved by the Y-12 Plant Technical Information Office for release to the public. Date: $5 \sqrt[9]{9} 97$ 


\section{Science Applications International Corporation}

contributed to the preparation of this document and should not be considered an eligible contractor for its review. 


\section{DISClamMER}

Portions of this document may be illegible in electronic image products. Images are produced from the best available original document. 


\section{Health and Safety Plan for Phase II of the Bear Creek Valley \\ Treatability Study Oak Ridge Y-12 Plant, Oak Ridge, Tennessee}

Date Issued-May 1997

Prepared by

Science Applications International Corporation

Oak Ridge, Tennessee

under subcontract 78B-99069C

Prepared for the

U.S. Department of Energy

Office of Environmental Management under budget and reporting code EW 20 "

Environmental Management Activities at the

OAK RIDGE Y-12 PLANT

Oak Ridge, Tennessee 37831

managed by

LOCKHEED MARTIN ENERGY SYSTEMS, INC.

for the

U.S. DEPARTMENT OF ENERGY

under contract DE-AC05-84OR21400 


\section{BEAR CREEK VALLEY TREATABILITY STUDY HEALTH AND SAFETY PLAN}
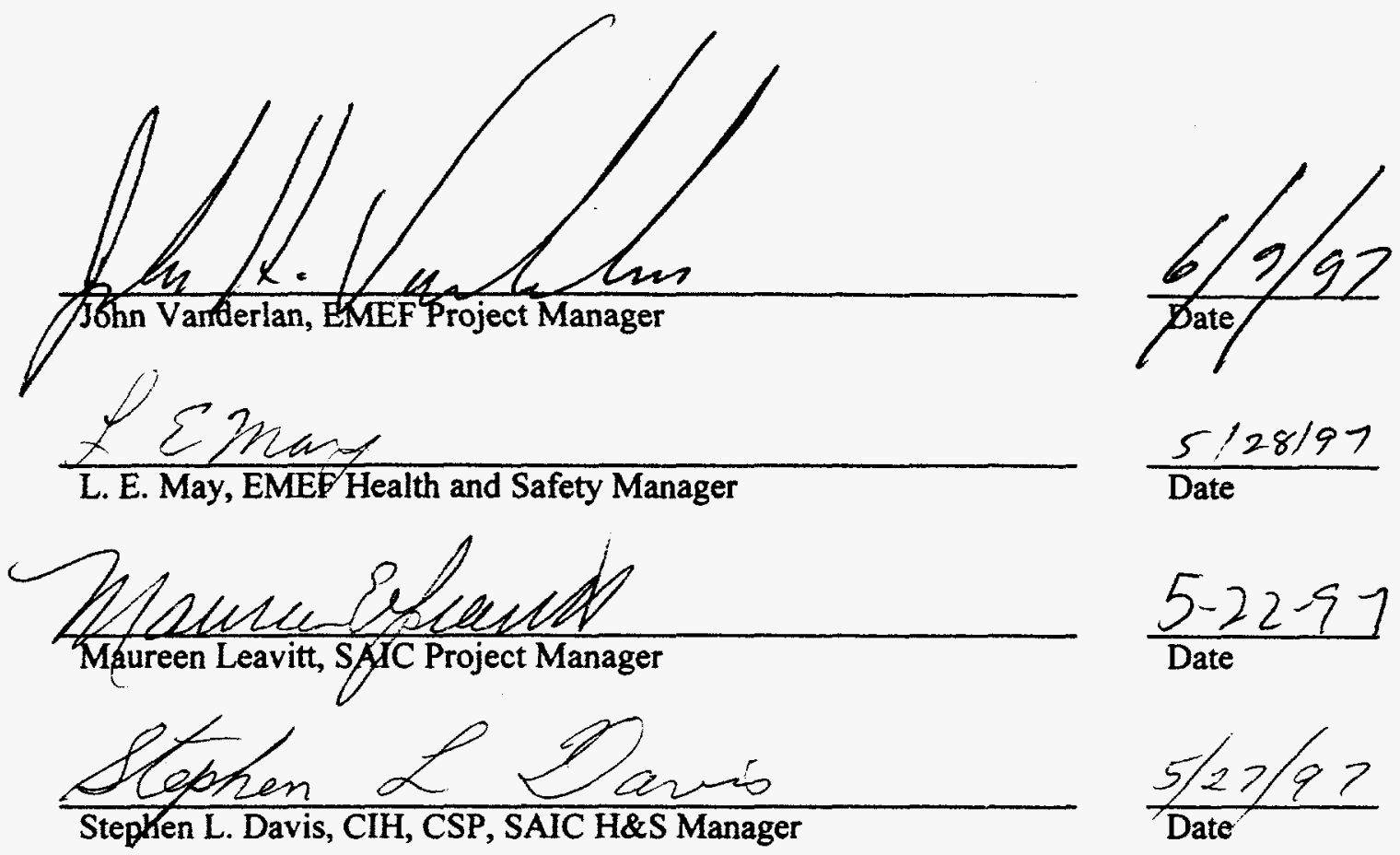

$\frac{5 / 28 / 97}{\text { Date }}$
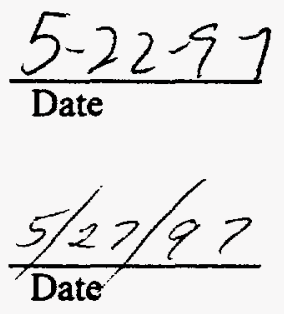



\section{PREFACE}

The Bear Creek Valley Treatability Study Health and Safety Plan was prepared under the Environmental Restoration Program to support the investigation of the practicality of the use of passive, in situ treatment systems to remove contaminants from the Bear Creek Valley Characterization area. This work was performed under Work Breakdown Structure (WBS) 1.1.02.41.10.34.20. This report will be used to direct investigations during Phase 2 . 


\section{CONTENTS}

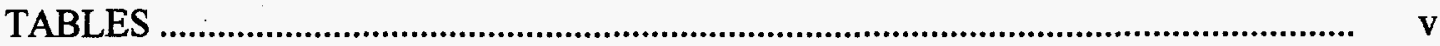

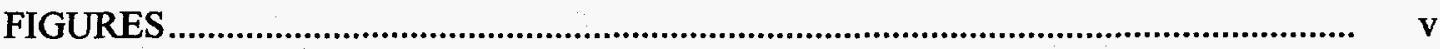

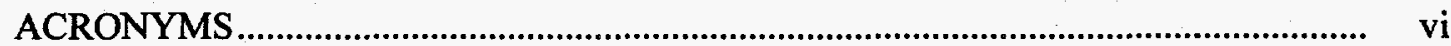

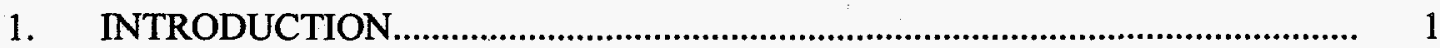

2. SITE HEALTH AND SAFETY PERSONNEL ....................................................

2.1 EMEF PROJECT MANAGER RESPONSIBILITIES ................................. 1

2.2 EMEF HEALTH AND SAFETY MANAGER .............................................. 2

2.3 SAIC PROJECT MANAGER ..................................................................... 3

2.4 SAIC FIELD OPERATIONS MANAGER .................................................. 3

2.5 SAIC SITE HEALTH AND SAFETY OFFICER ...................................... 3

2.6 FIELD PROJECT PERSONNEL ............................................................. 5

3. SAFETY RISK ANALYSIS .......................................................................... 5

3.1 TASK-SPECIFIC HAZARD EVALUATIONS ....................................... 12

3.1.1 Water Sampling ............................................................................ 12

3.1.2 Sample Preparation .................................................................... 12

3.1.3 Decontamination of Field Equipment ............................................... 13

3.2 GENERAL HAZARD CONTROLS....................................................... 13

3.2.1 Temperature Extremes .................................................................... 13

3.2.2 Animal Hazards and Vectors........................................................... 14

3.2.3 Illumination ..................................................................................... 14

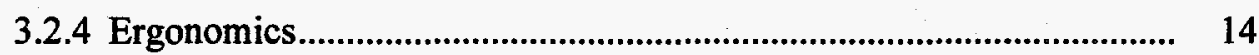

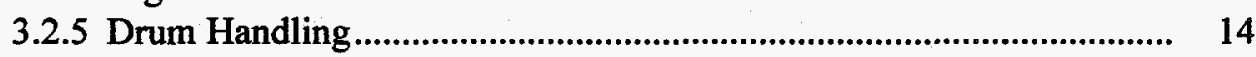

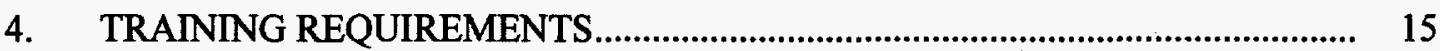

5. PERSONAL PROTECTIVE EQUIPMENT ........................................................... 17

$5.1 \quad$ UPGRADE OR DOWNGRADE OF PPE ................................................. 18

5.2 SITE PPE LEVELS............................................................................... 18

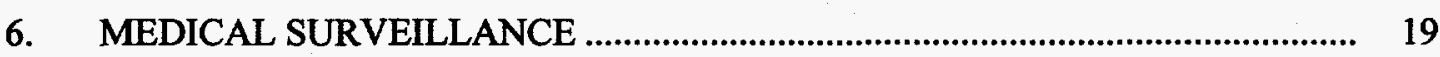

6.1 EMPLOYEES COVERED....................................................................... 19

6.2 FREQUENCY OF EXAM ..................................................................... 19

6.3 MEDICAL EXAM CONTENT …............................................................ 20

6.4 MEDICAL SURVEILLANCE FOR RESPIRATOR USERS ...................... 21

6.5 MEDICAL SUPPORT ........................................................................ 21

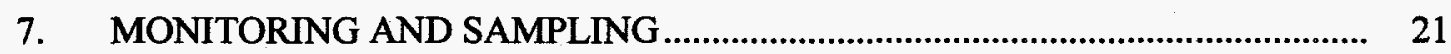

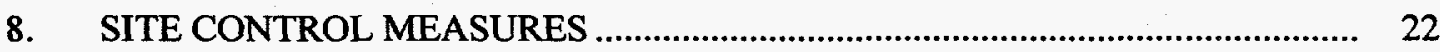

8.1 PROJECT-SPECIFIC SITE CONTROL ................................................. 22

8.2 PERMITS AND PASSES .................................................................... 23

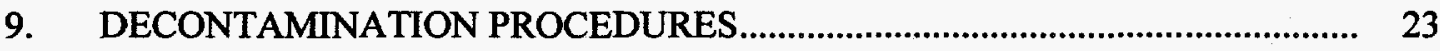


9.1 PERSONNEL DECONTAMINATION ....................................................... 23

9.2 EQUIPMENT DECONTAMINATION ...................................................... 23

9.3 DECONTAMINATION EQUIPMENT AND SUPPLIES .......................... 23

10. SITE OPERATING PROCEDURES............................................................ 24

10.1 SAFETY EQUIPMENT AND SITE COMMUNICATIONS ....................... 25

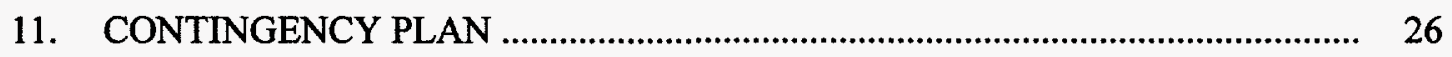

11.1 EMERGENCY PHONE NUMBERS....................................................... 26

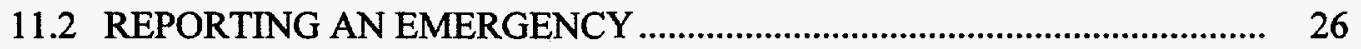

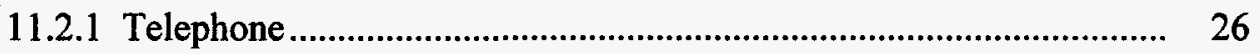

11.2.2 Fire Alarm Pull Boxes............................................................ 27

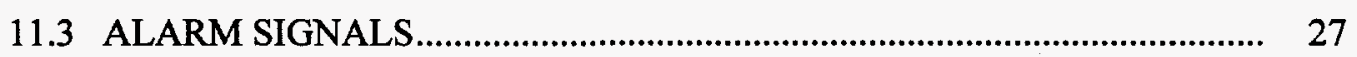

11.4 EVACUATION PROCEDURES ............................................................. 27

11.5 EMERGENCY EQUIPMENT ............................................................... 27

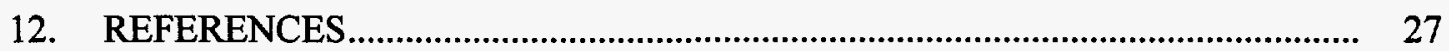




\section{TABLES}

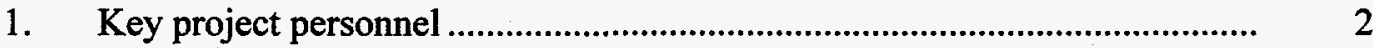

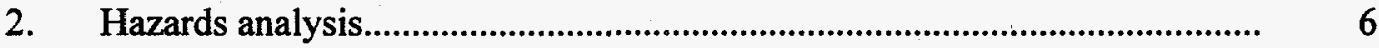

3. Potential chemical/radiological contaminants ............................................. 10

4. Training requirements.............................................................................. 16

5. Site monitoring action limits .............................................................. 21

\section{FIGURE}

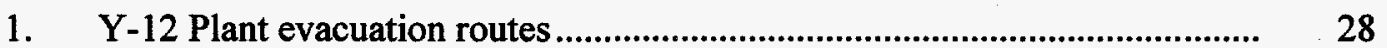




\section{ACRONYMS}

\begin{tabular}{|c|c|}
\hline ALI & annual limit on uptake \\
\hline ANSI & American National Standards Institute \\
\hline ASTM & American Society for Testing and Materials \\
\hline $\mathrm{BCV}$ & Bear Creek Valley \\
\hline CNS & central nervous system \\
\hline CVS & cardiovascular system \\
\hline DOT & U.S. Department of Transportation \\
\hline EMEF & Environmental Management and Enrichment Facilities \\
\hline Energy Systems & Lockheed Martin Energy Systems, Inc. \\
\hline EPA & Environmental Protection Ágency \\
\hline FCO & field change order \\
\hline FP & flash point \\
\hline GET & General Employee Training \\
\hline $\mathrm{H} \& \mathrm{~S}$ & health and safety \\
\hline HASP & Health and Safety Plan \\
\hline HP & Health Physics \\
\hline IDLH & immediately dangerous to life and health \\
\hline IH & Industrial Hygiene \\
\hline IP & ionization potential \\
\hline IS & Industrial Safety \\
\hline LEL & lower explosive limit \\
\hline MSDS & Material Safety Data Sheet \\
\hline NA & not applicable \\
\hline $\mathrm{NE}$ & not established \\
\hline OJT & on the job training \\
\hline ORR & Oak Ridge Reservation \\
\hline OSHA & Occupational Safety and Health Administration \\
\hline PEL & permissible exposure limit \\
\hline PID & photoionization detector \\
\hline PPE & personal protective equipment \\
\hline ppm & parts per million \\
\hline RCRA & Resource Conservation and Recovery Act \\
\hline RWP & Radiological Work Permits \\
\hline SAIC & Science Applications International Corporation \\
\hline SARA & Superfund Amendments and Reauthorization Act \\
\hline SHSO & Site Health and Safety Officer \\
\hline STEL & short-term exposure limit \\
\hline TLV & threshold limit value \\
\hline UEL & upper explosive limit \\
\hline VOC & volatile organic compound \\
\hline WBGT & wet bulb globe thermometer \\
\hline
\end{tabular}





\section{INTRODUCTION}

This Health and Safety Plan (HASP) addresses the health and safety (H\&S) concerns and requirements for the Bear Creek Valley (BCV) Treatability Study at the Oak Ridge Y-12 Plant. Samples will be collected from effluent following treatment tests of extraction columns, algal mats, and mature wetlands supplied by surface water locations and existing groundwater monitoring well locations. The project Sampling and Analysis Plan addresses the project description, technical objectives, procedures, and planned work activities in greater detail.

It is the responsibility of the project managers, field manager, and site health and safety officer (SHSO) to determine that the requirements of this HASP are sufficiently protective. If it is determined that the requirements of this HASP are not sufficiently protective, a field change order(s) (FCO) will be prepared. FCOs will include a completed job hazard analysis (see Table 3) or similar worksheet to ensure complete hazard assessment. FCOs must be approved by the Environmental Management and Enrichment Facilities (EMEF) project manager, EMEF H\&S manager, subcontractor project or field manager, and subcontractor H\&S representative. As a minimum, FCOs will be prepared if additional tasks will be performed or if contaminant exposure is anticipated.

\section{SITE HEALTH AND SAFETY PERSONNEL}

This project is being conducted under the direction of the EMEF Program. Table 1 shows key personnel and affiliations for this project. The roles of key H\&S personnel and field personnel are described in the following sections.

\subsection{EMEF PROJECT MANAGER RESPONSIBILITIES}

The EMEF Project Manager or designee is responsible for managing and coordinating project field activities. Responsibilities include the following:

- coordinating the support personnel required for field activities and ensuring that these personnel have received appropriate training;

- notifying the Plant Shift Supervisors of plans and scheduled activities for each site;

- obtaining any required permits and documentation;

- maintaining all training records of individuals involved in site activities;

- overseeing field activities;

- ensuring adherence to this HASP throughout the field operations; 
Table 1. Key project personnel

\begin{tabular}{llc}
\hline Responsibility & Name & Telephone \\
\hline EMEF Project Manager & John Vanderlan & $574-1660$ \\
EMEF H\&S Manager & Larry May & $576-4018$ \\
SAIC Project Manager & Maureen Leavitt & $481-8797$ \\
SAIC Field Operations Manager & Dan Thompson \\
SAIC SHSO & Mike Crenshaw & $481-4750$ \\
SAIC H\&S Manager & Steve Davis, CIH, & $481-4767$ \\
& CSP & $481-4755$ \\
\hline
\end{tabular}

- determining the intended use of data; and

- ensuring that any appropriate safety documentation (e.g., Safety Work Permits, safety reviews, safety inspections) is prepared for this project.

\subsection{EMEF HEALTH AND SAFETY MANAGER}

The EMEF H\&S Manager will provide appropriate technical oversight and field oversight as required to verify safe project execution. Specific activities include the following:

- reviewing appropriate project documents;

- reviewing and approving project plans before site activities begin;

- providing technical oversight;

- on-site auditing for compliance with federal, state, and facility regulations, standards, and orders;

- coordinating additional Lockheed Martin Energy Systems, Inc. (Energy Systems) H\&S support, as necessary; and

- interpreting federal, state, and facility regulations, standards, and orders. 


\subsection{SAIC PROJECT MANAGER}

The Science Applications International Corporation (SAIC) Project Manager is responsible for overall project execution. The responsibilities of the Project Manager include the following:

- coordinating with EMEF personnel, including project manager and field coordinator manager;

- ensuring that all changes to this project (i.e., operational, procedural, maintenance, etc.) are reviewed for Unreviewed Safety Questions [per DOE Order 5480.21, Unreviewed Safety Questions (USQ)];

- maintaining auditable project documentation of all required records;

- ensuring that a qualified SHSO is designated; and

- maintaining a current copy of the HASP.

\subsection{SAIC FIELD OPERATIONS MANAGER}

The SAIC Field Operations Manager will oversee the field activities associated with this project and will be responsible for site accessibility, safety, and quality assurance and will delegate further responsibilities to other members of the sampling team. He/she is responsible for implementing and enforcing the field requirements of this HASP. Specific responsibilities of the SAIC Field Operations Manager are listed below:

- ensuring that all personnel on site follow the requirements of the HASP;

- coordinating on-site operations, including logistics;

- interfacing with plant and project personnel;

- maintaining auditable project documentation of all required records;

- maintaining a current copy of the HASP;

- controlling visitor access to field operations; and

- minimizing the number of personnel and the amount of equipment in the exclusion zone, but only to the extent consistent with safe site operations.

\subsection{SAIC SITE HEALTH AND SAFETY OFFICER}

The SHSO makes H\&S decisions and performs specific H\&S activities. The SHSO has primary responsibility for the following: 
- verifying compliance with the HASP and reporting to the Project Manager and/or appropriate H\&S department such as Industrial Hygiene (IH), Industrial Safety (IS), or Health Physics (HP);

- stopping work and/or upgrading protective $\mathrm{H} \& \mathrm{~S}$ measures [e.g., personal protective equipment (PPE), respiratory protection, etc.] in the event that uncontrolled or unanticipated $H \& S$ hazards are encountered in the field;

- authorizing the return to work following resolution of the H\&S hazard(s) or other "stop-work" issues;

- ensuring that personnel have access to this HASP and are aware of its provisions, are instructed in safe work practices, and are familiar with potential hazards and planned emergency procedures prior to initiation of site activities;

- maintaining on-site copies of Material Safety Data Sheets (MSDSs) for applicable materials used at the site;

- verifying that all on-site personnel have received the training listed in the Training Requirements section of this HASP and maintaining on-site copies of current training records;

- establishing and making available at the site appropriate means of communication, along with telephone numbers and radio communication information;

- ensuring that all monitoring equipment is calibrated (or calibration checked) prior to each day's use and is operating according to the manufacturer's specifications and instructions;

- recording calibration results and any maintenance notes in the field logbook;

- verifying that all required project permits have been generated and that signed copies are available on site before field work begins;

- ensuring that all chemicals not in their original containers are labeled properly;

- ensuring that personnel stay out from under overhead work and temporarily suspended equipment;

- ensuring that appropriate monitoring for potential on-site contaminants during field operations is conducted;

- conducting a pre-entry H\&S briefing and appropriate follow-on briefings, as required, to address pertinent H\&S information (e.g., known or suspected hazards, safety precautions, emergency actions) including the contents of this HASP;

- documenting field changes to this HASP in the project field logbook; and

- controlling visitor access to the exclusion and contamination reduction zones. 


\subsection{FIELD PROJECT PERSONNEL}

Project personnel involved in on-site operations will have the following responsibilities:

- performing only those tasks they believe they can do safely;

- stopping all work activities in the event of any of the following conditions:

- inadequate H\&S controls,

- H\&S controls not being implemented,

- Radiological Control Hold Pcint not being satisfied, or

- any imminent danger associated with work activities;

- notifying the SHSO of any medical conditions (e.g., allergies, diabetes, pregnancy) that may require special considerations;

- abiding by a buddy system so that each on-site worker is responsible for keeping track of his or her partner in the event of an emergency;

- following radiological controls and practices appropriate for site operations as instructed by HP technicians;

- wearing personal exposure monitoring devices during on-site activities as required by Radiological Work Permits (RWP), signs, procedures, this HASP, or by radiological or IH personnel;

- immediately reporting the loss, damage, or unexpected exposure of personal monitoring devices or any off-scale readings to the appropriate H\&S department;

- immediately notifying the SHSO of symptoms or signs of exposure potentially related to any chemical, physical, or biological hazards present at the site; and

- performing all field activities in accordance with As Low As Reasonably Achievable program requirements.

\section{SAFETY RISK ANALYSIS}

The purpose of the site task hazard analysis is to identify and assess potential hazards that site personnel might encounter and to prescribe required methods of hazard control. Table 2 summarizes the anticipated site and operational hazards and identifies appropriate control measures to be used. Table 3 lists the potential site contaminants and any hazardous chemicals that will be used on the site. This list of potential site contaminants was derived from existing characterization and monitoring data. Contaminants were selected for inclusion in Table 3 using the following criteria: 
Table 2. Hazards analysis

\section{Safety and health hazards}

\section{Effluent Sampling}

General hazards

(e.g., slips, falls, lifting, moving equipment)

Chemical contaminants and preservatives (See Table 3)

Radioactive contamination

(if detected on site)

(See Table 3)

Temperature extremes

Animal hazards

(e.g., bees, ticks, wasps, snakes, rodents)
Minimum PPE for task (level D):

Field work clothes at Y-12 Plant

Safety glasses

Protective footwear, ANSI Z41-1991

Disposable latex gloves or disposable nitrile gloves

(as appropriate)

Determination of physical hazards prior to materials(s)

handling

Good ergonomic work practices

Level D PPE (including safety glasses, chemical resistant gloves)

Minimize contact

Wash hands before eating or drinking

$15 \mathrm{~min}$ eyewash within $100 \mathrm{ft}$ when pouring corrosives

Comply with relevant RWPs

Minimize contact

Decontamination (as required)

Administrative controls

Hydration and acclimatization of workers

Appropriate clothing for ambient site conditions

(See Sect. 3.2.1)

Work clothing and boots

Insect repellent, as appropriate

(See Sect. 3.2.2)
Visual survey of site for obstacles prior to and

during container lifting or maneuvering

Verification by SHSO

Monitoring

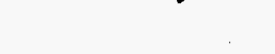

Survey for radioactivity and/or air sampling, as appropriate

Personal dosimetry

Observation of workers

Pulse rate monitoring and/or WBGT

measurements, as appropriate

Visual inspection of site

Self-monitoring for and reporting of bites/stings 
Table 2. (continued)

\begin{tabular}{ll}
\hline Safety and health hazards & Controls \\
\hline & \multicolumn{1}{c}{ Surface Water and Groundwater Sampling } \\
& Minimum PPE for task (level D): \\
$\begin{array}{l}\text { General hazards } \\
\text { (e.g., slips, falls, lifting, moving equipment) }\end{array}$ & Field work clothes at Y-12 Plant \\
& Safety glasses \\
& Protective footwear, ANSI Z41-1991 \\
& Disposable latex gloves or disposable nitrile gloves \\
& (as appropriate) \\
& Determination of physical hazards prior to materials(s) \\
& handling \\
& Good ergonomic work practices \\
& Level D PPE \\
Chemical contaminants & Minimize contact \\
(See Table 3) & Decontamination (as required) \\
& Comply with relevant RWPs \\
Radioactive contamination & Minimize contact \\
(See Table 3) & Decontamination (as required) \\
& Administrative controls \\
Temperature extremes & Hydration and acclimatization of workers \\
& Appropriate clothing for ambient site conditions \\
& (See Sect. 3.2.1) \\
& Work clothing and boots \\
Building hazards & Insect repellent, as appropriate \\
(See Sect. 3.2.2) \\
(e.g., bees, ticks, wasps, snakes, rodents) & Coordinate with building manager \\
& Follow building requirements \\
&
\end{tabular}

Monitoring

Survey for radioactivity and/or air sampling, as

Visual survey of site for obstacles prior to and during container lifting or maneuvering appropriate

Personal dosimetry

Observation of workers

Pulse rate monitoring and/or WBGT

measurements, as appropriate

Visual inspection of site

Self-monitoring for and reporting of bites/stings

Visual survey for hazards, such as slippery surfaces, tripping hazards, confined spaces 
Table 2. (continued)

\section{Water Sample Preparation} (sample packaging, addition of preservatives)

General hazards

(e.g., slips, falls, lifting, moving equipment)

Chemical contaminants

(See Table 3)

Radioactive contamination

(See Table 3)

Temperature extremes

Animal hazards

(e.g., bees, ticks, wasps, snakes, rodents)
Minimum PPE for task (level D):

Field work clothes at Y-12 Plant

Safety glasses

Protective footwear, ANSI Z41-1991

Disposable latex gloves or disposable nitrile gloves (as appropriate)

Determination of physical hazards prior to materials(s)

handling

Good ergonomic work practices

\section{Level D PPE}

Minimize contact

Wash hands before eating or drinking

Comply with relevant RWPs

Decontamination (as required)

Administrative controls

Hydration and acclimatization of workers

Appropriate clothing for ambient site conditions

(See Sect. 3.2.1)

Work clothing and boots

Insect repellent, as appropriate

(See Sect. 3.2.2)
Minimize contact
Visual survey of site for obstacles prior to and during container lifting or maneuvering

Survey for radioactivity and/or air sampling, as appropriate

Personal dosimetry

Observation of workers

Pulse rate monitoring and/or WBGT

measurements, as appropriate

Visual inspection of site

Self-monitoring for and reporting of bites/stings 
Table 2. (continued)

Decontamination of Equipment

(Liquinox wash, water rinse is propanol rinse)

General hazards

(e.g., slips, falls, lifting, moving equipment)

Release of contaminants to the environment during decontamination activities

Chemical contaminants and preservatives (See Table 3)

Temperature controls

Fire

PPE: personal protective equipment

RWP: Radiological Work Permits

SHSO: Site Health and Safety Officer

WBGT: Wet Bulb Globe Temperature
Visual survey of site for obstacles prior to and during container lifting or maneuvering

for task (level D):

Field work clothes at Y-12 Plant

Safety glasses

Protective footwear, ANSI Z41-1991

Disposable latex gloves or disposable nitrile gloves

(as appropriate)

Containment systems appropriate for level(s) of contaminants(s)

Level D PPE (safety glasses, chemical resistant gloves)

Minimize contact

Wash hands before eating or drinking

Administrative controls

Hydration and acclimatization of workers

Appropriate clothing for ambient site conditions

(See Sect. 3.2.1)

Fire extinguisher within $25 \mathrm{ft}$
Monitoring methods appropriate for level(s) of contaminant(s)

None

Observation of workers

Pulse rate monitoring and/or WBGT

measurements, as appropriate

Verification by SHSO 
Table 3. Potential chemical/radiological contaminants

\begin{tabular}{|c|c|c|c|c|}
\hline Contaminant & $\begin{array}{c}\text { TLV/PEL/STEL/ } \\
\text { IDLH/ALI } \\
\end{array}$ & $\begin{array}{c}\text { Potential health effects/ } \\
\text { target organs }\end{array}$ & Chemical and physical properties & $\begin{array}{c}\text { Exposure } \\
\text { route(s) }\end{array}$ \\
\hline 1,1-Dichloroethane & $\begin{array}{l}\text { PEL: } 100 \mathrm{ppm} \\
\text { TLV: } 100 \mathrm{ppm}, \mathrm{A} 4 \\
\text { IDLH: } 3000 \mathrm{ppm}\end{array}$ & $\begin{array}{l}\text { CNS depression; skin, liver, and } \\
\text { kidney damage; irritant }\end{array}$ & $\begin{array}{l}\text { Colorless oily liquid with a } \\
\text { chloroform-like odor, } \\
\text { FP: } 2^{\circ} \mathrm{F} \text {, IP: } 11.06 \mathrm{eV} \text {, } \\
\text { LEL: } 5.4 \%\end{array}$ & $\begin{array}{l}\text { Inhalation } \\
\text { Ingestion } \\
\text { Contact }\end{array}$ \\
\hline 1,2-Dichloroethene & $\begin{array}{l}\text { TLV: } 200 \mathrm{ppm} \\
\text { STEL: NA } \\
\text { IDLH: } 1000 \mathrm{ppm}\end{array}$ & CNS depression; irritant & $\begin{array}{l}\text { Colorless liquid, FP: } 36^{\circ} \mathrm{F} \text {, } \\
\text { IP: } 9.65 \mathrm{eV}\end{array}$ & $\begin{array}{l}\text { Inhalation } \\
\text { Ingestion } \\
\text { Contact }\end{array}$ \\
\hline $\begin{array}{l}\text { Hydrochloric acid (used to } \\
\text { preserve some water } \\
\text { samples) }\end{array}$ & $\begin{array}{l}\text { TLV: C5 ppm } \\
\text { STEL: NA } \\
\text { IDLH: } 50 \mathrm{ppm}\end{array}$ & Irritant; causes tissue damage & $\begin{array}{l}\text { Liquid with pungent odor, } \\
\text { FP: NA, IP: } 12.74 \mathrm{eV}\end{array}$ & $\begin{array}{l}\text { Inhalation } \\
\text { Ingestion } \\
\text { Contact }\end{array}$ \\
\hline $\begin{array}{l}\text { Isopropyl alcohol (used for } \\
\text { decontamination) }\end{array}$ & $\begin{array}{l}\text { IDLH: } 2000 \mathrm{ppm} \\
\text { TLV: } 400 \mathrm{ppm}\end{array}$ & $\begin{array}{l}\text { Irritant to eyes, nose, throat; can } \\
\text { cause dizziness }\end{array}$ & $\begin{array}{l}\text { Colorless liquid with the odor of } \\
\text { rubbing alcohol, FP: } 53^{\circ} \mathrm{F} \text {, } \\
\text { IP: } 10.10 \mathrm{eV}\end{array}$ & $\begin{array}{l}\text { Inhalation } \\
\text { Ingestion } \\
\text { Contact }\end{array}$ \\
\hline $\begin{array}{l}\text { Liquinox (used for } \\
\text { decontamination) }\end{array}$ & NE & $\begin{array}{l}\text { Potential irritation of mucous } \\
\text { membranes }\end{array}$ & $\begin{array}{l}\text { Yellow liquid, odorless, completely } \\
\text { soluble in water, biodegradable }\end{array}$ & Ingestion \\
\hline $\begin{array}{l}\text { Nitric acid (used to } \\
\text { preserve some water } \\
\text { samples) }\end{array}$ & $\begin{array}{l}\text { TLV: } 2 \mathrm{ppm} \\
\text { STEL: } 4 \mathrm{ppm} \\
\text { IDLH: } 25 \mathrm{ppm}\end{array}$ & $\begin{array}{l}\text { Irritation of eyes, skin, mucous } \\
\text { membranes }\end{array}$ & $\begin{array}{l}\text { Liquid with pungent odor, } \\
\text { FP: NA, IP: } 11.95 \mathrm{eV}\end{array}$ & $\begin{array}{l}\text { Inhalation } \\
\text { Ingestion } \\
\text { Contact }\end{array}$ \\
\hline Sodium hydroxide $(\mathrm{NaOH})$ & $\begin{array}{l}\text { IDLH: } 10 \mathrm{mg} / \mathrm{m}^{3} \\
\text { TLV: } \mathrm{C} 2 \mathrm{mg} / \mathrm{m}^{3}\end{array}$ & $\begin{array}{l}\text { Irritation to eyes, nose; can cause } \\
\text { temporary loss of hair }\end{array}$ & $\begin{array}{l}\text { Colorless to white, odorless solid } \\
\text { (flakes, beads, granular form), } \\
\text { FP: NA, IP: NA }\end{array}$ & $\begin{array}{l}\text { Inhalation } \\
\text { Ingestion } \\
\text { Contact }\end{array}$ \\
\hline Technetium-99 & $\begin{array}{l}\text { Oral: } 1 \mathrm{E} 08 \mathrm{~Bq} \\
\text { Class D: } 2 \mathrm{E} 08 \mathrm{~Bq} \\
\text { Class W: } 2 \mathrm{E} 07 \mathrm{~Bq} \\
\text { Class Y: (not listed) }\end{array}$ & Carcinogen, mutagen, teratogen & $\begin{array}{l}\text { Variable, depends on compounds } \\
\text { present }\end{array}$ & $\begin{array}{l}\text { Inhalation } \\
\text { Ingestion } \\
\text { Absorption } \\
\text { Injection }\end{array}$ \\
\hline
\end{tabular}


Table 3. (continued)

\begin{tabular}{|c|c|c|c|c|}
\hline Contaminant & $\begin{array}{c}\text { TLV/PEL/STEL/ } \\
\text { IDLH/ALI }\end{array}$ & $\begin{array}{c}\text { Potential health effects/ } \\
\text { target organs }\end{array}$ & Chemical and physical properties & $\begin{array}{c}\text { Exposure } \\
\text { route(s) }\end{array}$ \\
\hline Tetrachloroethene & $\begin{array}{l}\text { TLV: } 25 \mathrm{ppm}, \mathrm{A3} \\
\text { STEL: } 100 \mathrm{ppm}, \mathrm{A3} \\
\text { IDLH: Ca }(150 \mathrm{ppm})\end{array}$ & $\begin{array}{l}\text { Potential human carcinogen; irritant; } \\
\text { causes dizziness, liver damage }\end{array}$ & Colorless liquid, FP: NA, IP: $9.32 \mathrm{eV}$ & $\begin{array}{l}\text { Inhalation } \\
\text { Absorption } \\
\text { Ingestion } \\
\text { Contact }\end{array}$ \\
\hline $\begin{array}{l}\text { 1,1,1-Trichloroethane } \\
\text { (methyl chloroform) }\end{array}$ & $\begin{array}{l}\text { TLV: } 350 \mathrm{ppm}, \mathrm{A4} \\
\text { STEL: } 450 \mathrm{ppm} \\
\text { IDLH: } 700 \mathrm{ppm}\end{array}$ & $\begin{array}{l}\text { Suspect carcinogen; CNS, CVS, } \\
\text { eyes, skin irritant; causes headaches, } \\
\text { drowsiness, and unconsciousness; } \\
\text { impairs judgment }\end{array}$ & $\begin{array}{l}\text { Colorless liquid, mild chloroform-like } \\
\text { odor, LEL: } 7 \% \text {, UEL: } 12.5 \% \text {, IP: } \\
11.00 \mathrm{eV} \text {, FP: NE }\end{array}$ & $\begin{array}{l}\text { Inhalation } \\
\text { Ingestion } \\
\text { Contact }\end{array}$ \\
\hline Trichloroethene & $\begin{array}{l}\text { TLV: } 50 \mathrm{ppm}, \mathrm{A5} \\
\text { STEL: } 100 \mathrm{ppm}, \text { A5 } \\
\text { IDLH: Ca }(1000 \mathrm{ppm})\end{array}$ & $\begin{array}{l}\text { Carcinogen; causes headaches, } \\
\text { vertigo, visual disturbance, tremors; } \\
\text { irritant; causes dermatitis }\end{array}$ & $\begin{array}{l}\text { Colorless liquid, mild chloroform-like } \\
\text { odor, LEL: } 8 \% \text {, UEL: } 10.5 \% \text {, IP: } 9.45 \\
\text { eV, FP: } 90^{\circ} \mathrm{F}\end{array}$ & $\begin{array}{l}\text { Inhalation } \\
\text { Absorption } \\
\text { Ingestion } \\
\text { Contact }\end{array}$ \\
\hline Uranium-234 & $\begin{array}{l}\text { Oral: } 4 \mathrm{E} 05 \mathrm{~Bq} \\
\text { Class D: } 5 \mathrm{E} 04 \mathrm{~Bq} \\
\text { Class W: } 3 \mathrm{E} 04 \mathrm{~Bq} \\
\text { Class Y: } 1 \mathrm{E} 03 \mathrm{~Bq}\end{array}$ & $\begin{array}{l}\text { Carcinogen, mutagen, teratogen; } \\
\text { causes renal damage }\end{array}$ & $\begin{array}{l}\text { Variable, depends on compounds } \\
\text { present }\end{array}$ & $\begin{array}{l}\text { Inhalation } \\
\text { Ingestion } \\
\text { Absorption } \\
\text { Injection }\end{array}$ \\
\hline Uranium-235 & $\begin{array}{l}\text { Oral: } 5 \mathrm{E} 05 \mathrm{~Bq} \\
\text { Class D: } 5 \mathrm{E} 04 \mathrm{~Bq} \\
\text { Class W: } 3 \mathrm{E} 04 \mathrm{~Bq} \\
\text { Class Y: } 2 \mathrm{E} 03 \mathrm{~Bq}\end{array}$ & $\begin{array}{l}\text { Carcinogen, mutagen, teratogen; } \\
\text { causes renal damage }\end{array}$ & $\begin{array}{l}\text { Variable, depends on compounds } \\
\text { present }\end{array}$ & $\begin{array}{l}\text { Inhalation } \\
\text { Ingestion } \\
\text { Absorption } \\
\text { Injection }\end{array}$ \\
\hline Uranium-238 & $\begin{array}{l}\text { Oral: } 5 \mathrm{E} 05 \mathrm{~Bq} \\
\text { Class D: } 5 \mathrm{E} 04 \mathrm{~Bq} \\
\text { Class W: } 6 \mathrm{E} 07 \mathrm{~Bq} \\
\text { Class Y: } 2 \mathrm{E} 03 \mathrm{~Bq}\end{array}$ & $\begin{array}{l}\text { Carcinogen, mutagen, teratogen; } \\
\text { causes renal damage }\end{array}$ & $\begin{array}{l}\text { Variable, depends on compounds } \\
\text { present }\end{array}$ & $\begin{array}{l}\text { Inhalation } \\
\text { Ingestion } \\
\text { Absorption } \\
\text { Injection }\end{array}$ \\
\hline Vinyl chloride & $\begin{array}{l}\text { TLV: } 5 \text { ppm, Al } \\
\text { STEL: NA }\end{array}$ & $\begin{array}{l}\text { Carcinogen; causes weakness, liver } \\
\text { damage }\end{array}$ & $\begin{array}{l}\text { Colorless flammable gas (liquid below } \\
7^{\circ} \mathrm{F} \text { ), FP: NA (gas), LEL: } 3.6 \% \text {, IP: } \\
9.99 \mathrm{eV}\end{array}$ & $\begin{array}{l}\text { Inhalation } \\
\text { Contact }\end{array}$ \\
\hline
\end{tabular}

Table information from the American Conference of Industrial Hygienists' 1996 Threshold Limit Values for Chemical Substances and Physical Agents and Biological Exposure Indices, the National Institute of Occupational Safety and Health Pocket Guide to Chemical Hazards (June 1994), and the International Commission on Radiological Protection Publication 30.

ALI: annual limit on intake

$$
\text { C: ceiling }
$$

CNS: central nervous system

CVS: cardiovascular system

FP: flash point

IDLH: immediately dangerous to life and health

IP: ionization potential

\author{
LEL: lower explosive limit \\ NA: not applicable \\ NE: not established \\ PEL: permissible exposure limit \\ STEL: short-term exposure limit \\ TLV: threshold limit value \\ UEL: upper explosive limit
}

A2 suspected human carcinogen
A1 confirmed human carcinogen
A3 animal carcinogen
A4 not classified as a human carcinogen
A5 not suspected as a human carcinogen 
- having a permissible exposure limit (PEL) or a threshold limit value (TLV) of $\leq 200$ parts per million (ppm) or $\leq 5 \mathrm{mg} / \mathrm{m}^{3}$; and

- being known or suspected to be present at the site in significant quantity, based on site historical records; or

- being detected in previous groundwater samples at concentrations greater than drinking water standards.

Examples of previously detected contaminants in groundwater and their corresponding maximum concentrations are 1,2-dichloroethene $(3000 \mu \mathrm{g} / \mathrm{L})$, tetrachloroethene $(910 \mu \mathrm{g} / \mathrm{L})$, trichloroethene $(15000 \mu \mathrm{g} / \mathrm{L})$, and vinyl chloride $(480 \mu \mathrm{g} / \mathrm{L})$. Other known contaminants include heavy metals and isotopes of uranium and technitium. Based on the low maximum concentrations and previous real-time instrument monitoring at groundwater sampling locations for organic vapors and radioactivity, no exposures greater than Occupational Safety and Health Administration (OSHA) action limits are anticipated.

\subsection{TASK-SPECIFIC HAZARD EVALUATIONS}

\subsubsection{Water Sampling}

The project will use multiple test media (exchange resins, iron oxide floculants and peat moss in filtration columns, algal mats, wetlands) that will operate simultaneously. Groundwater from selected wells will be used to supply the filtration columns and algal mats. Surface water samples from the wetlands will be collected with dippers or similar implements.

Based on previously obtained sample data, the risk of chemical or radiological exposure is minimal. In a real-time assessment of wells using a photoionization detector (PID), the presence of volatile organic compounds (VOCs) was detected in a limited number of the wells. However, no VOCs were measured in the worker breathing zone (i.e., $3 \mathrm{ft}$ above the opening or $14 \mathrm{in}$. in front of worker's shoulder). Therefore, it is unlikely that any worker exposure will occur during the work activities planned for this project.

Historical site data indicate that radiation exposure or radiological contamination of site personnel from groundwater or surface water is not a concern within the scope of this project. Project personnel will comply with all relevant RWPs. Although no radiological exposure is anticipated during planned site activities, an HP technician may periodically monitor site operations and site equipment for radiological contamination.

\subsubsection{Sample Preparation}

Water samples will be prepared for shipment and analysis through a variety of techniques. Small sample quantities and screening prior to sampling will ensure that water contaminants will pose no significant hazard during this preparation. Sample preservatives for some types of samples will pose a corrosive hazard to the eyes and skin. The sample preservatives (hydrochloric acid, nitric acid, etc.) will be used in quantities of a few milliliters. To prevent eye and skin contact, gloves and safety glasses will be worn when these preservatives are handled. A 
15-min emergency eyewash will also be kept immediately available (within $100 \mathrm{ft}$ ) when corrosive materials are being poured.

\subsubsection{Decontamination of Field Equipment}

All reusable field equipment that comes into direct or incidental contact with water will be decontaminated before reuse to prevent cross-contamination. Decontamination activities include potential exposure to site contaminants as well as to detergent and isopropyl alcohol. The detergent poses a minimal eye hazard. Isopropyl alcohol is irritating to the eyes and is a flammable liquid. Safety glasses and gloves will be worn when using these materials. Isopropyl alcohol will only be used in small quantities $(\leq 1 \mathrm{~L})$ to minimize the fire hazard. Decontamination of equipment is detailed in Chap. 9.

\subsection{GENERAL HAZARD CONTROLS}

In addition to the physical, chemical, and radiological hazards of the contaminants listed in Table 3, other types of potential hazards may be present on site. Personnel may encounter the hazards outlined below.

\subsubsection{Temperature Extremes}

Field activities conducted during the summer or winter pose a hazard because of temperature extremes. The physical demands placed on investigative personnel may be compounded by the use of protective clothing/equipment, moderate to heavy workloads, ambient air temperatures, relative humidity, and exposure to nonionizing radiation. The pre-entry briefing will include information on control measures for temperature extremes and the signs and symptoms of temperature-related health effects.

Two important factors will help personnel function in hot environments: acclimatization and fluid consumption. Acclimatization of workers to hot environmental conditions may require up to a week. During this period especially, workers should concentrate on maintaining a balanced diet, consuming plenty of fluids (while outside contaminated areas) throughout the day, and remaining aware of signs of heat-related illness. Headaches, dizziness, high body temperature, and increased heart rate are all early warning signals of heat stress. Work will be self-paced and any employee who experiences these symptoms is encouraged to take a break to allow adequate cooling. It is imperative that the SHSO be informed if a worker experiences these signs. All activities that take place at the investigative site require the use of a buddy system, and as field activities continue, all personnel should be apprised of their buddy's condition with respect to heat stress.

If necessary, in the judgment of the SHSO, a work/rest regimen will be instituted to help in combating heat-related disorders. Physiologic monitoring will be conducted if ambient temperatures exceed $75^{\circ} \mathrm{F}$. If physiologic monitoring is conducted, it will be conducted during rest periods to minimize the potential for chemical or radiological contamination. No employee may resume work following a break unless that employee's pulse rate is less than 100 beats/minute. 
Critical factors in preventing cold-stress disorders are adequate clothing and staying dry. The SHSO will ensure that individuals who become wet are moved to a sheltered, warm area quickly, and that personnel are appropriately dressed for site conditions. The SHSO will also ensure that a warm, sheltered area is immediately available.

\subsubsection{Animal Hazards and Vectors}

Field conditions may present a variety of animal hazards such as bees, ticks, poisonous snakes, and rodents. Wildlife can cause bodily injury to site personnel or may serve as vectors that transmit diseases. To reduce contact with animal hazards, employees will avoid heavy cover whenever possible and look for snakes or animals before disturbing potential habitats. Insect repellent may be applied to the lower body to prevent tick attachment. Employees will not handle or otherwise disturb wildlife.

\subsubsection{Illumination}

Outside operations will begin no earlier than $15 \mathrm{~min}$ after sunrise and conclude no later than $15 \mathrm{~min}$ before sunset. Because most activities will be conducted during daylight hours, field illumination measurements will not normally be required. However, if activities require outside work to be done at night, a minimum of $5 \mathrm{ft}-\mathrm{c}$ will be required.

\subsubsection{Ergonomics}

Potential hazards related to the interaction of personnel with their working environment may be present at this site. The primary ergonomic hazards that may exist are lifting heavy loads and physical obstacles associated with traversing rough ground. Personnel should always position themselves properly and lift from the legs when attempting to lift equipment. Each employee should rely on his/her buddy to assist in lifting loads that are too heavy for one person to properly lift and carry. Back strain, the most common ergonomic hazard in the field, can be easily avoided, provided the on-site workers always ask for assistance when they need it.

\subsubsection{Drum Handling}

Drums will be used to store and transport decontamination rinsate. All drum and container handling will comply with the following general requirements:

- Drums and containers will meet the appropriate U.S. Department of Transportation (DOT), OSHA, and U.S. Environmental Protection Agency (EPA) regulations for the wastes they contain.

- Site operations will be organized to minimize the amount of drum or container movement.

- Before moving drums or containers, all employees exposed to the transfer operations will be warned of the potential hazards of the drum or container contents.

- DOT-specified salvage drums or containers and suitable quantities of proper absorbent will be kept available and used in areas where spills, leaks, or ruptures may occur. 
- Where there is a reasonable possibility of flammable atmospheres being present, material handling equipment and hand tools will be of a type designed to prevent sources of ignition.

- Employees will not stand on or work from drums.

\section{TRAINING REQUIREMENTS}

All personnel who perform hands-on field work on this project must have received the following trairing:

- Energy Systems General Employee Training (GET),

- 40-h Hazardous Waste Operations Training,

- 8-h Refresher Training (as required),

- 8-h Hazardous Waste Site Supervisor Training (for supervisors and SHSOs supervisors),

- Radiological Worker Training II (for work in RAD-contaminated areas),

- Hazard Communication, and

- Site-Specific Training.

The following paragraphs provide brief descriptions of the relevant training. Additional information is provided in Table 4.

Energy Systems GET is required for all Energy Systems employees and any site visitor who visits the controlled area of an Energy Systems-operated site to perform work for more than 10 working days per year. This training addresses general site features and hazards, alarm signals, and evacuation.

Energy Systems Radiation Worker training (Rad Worker II) is required for unescorted entry into radiological contamination areas. This training is designed to enable attendees to work safely within a radiological control zone. It is $20 \mathrm{~h}$ in duration and includes donning/doffing, frisker, and general techniques for contamination control.

The 40-h Hazardous Waste Site Worker course is required for hands-on workers in the exclusion (contamination) or contamination reduction (buffer) zone, and anyone who will spend extended periods in the exclusion (contamination) or contamination reduction (buffer) zone. This course is mandated by the Hazardous Waste Operations and Emergency Response Standard (29 CFR 1910.120). It is designed to enable employees to operate safely on an uncontrolled hazardous waste site. Twenty-four hours of relevant field experience is required in conjunction with this training.

The 24-h Hazardous Waste Site course is required for occasional visitors to the exclusion (contamination) or contamination reduction (buffer) zones. This course is mandated by the Hazardous Waste Operations and Emergency Response Standard (29 CFR 1910.120). It is designed to enable employees to safely visit a hazardous waste site or work at a Resource Conservation and Recovery Act (RCRA) Treatment Storage or Disposal Facility. Eight hours of relevant field experience is required in conjunction with this course. 
Table 4. Training requirements

\begin{tabular}{|c|c|c|c|c|}
\hline Training & Worker & Supervisor & $\begin{array}{l}\text { Site visitor } \\
\text { (unescorted) }\end{array}$ & $\begin{array}{l}\text { Site visitor } \\
\text { (escorted) }\end{array}$ \\
\hline $\begin{array}{l}\text { General Employee Training } \\
(6.5 \mathrm{~h})\end{array}$ & $\sqrt{ }$ & $\sqrt{ }$ & $\sqrt{ }$ & $\sqrt{ }$ \\
\hline $\begin{array}{l}\text { Radiation Worker } \\
\text { (radiological contamination } \\
\text { areas) }\end{array}$ & $\sqrt{ }$ & $\sqrt{ }$ & $\sqrt{ }$ & $\mathrm{x}$ \\
\hline $\begin{array}{l}\text { Hazardous Waste } \\
(40 \mathrm{~h}, 24 \mathrm{~h} \text { OJT })\end{array}$ & $\sqrt{ }$ & $\sqrt{ }$ & $\mathbf{x}$ & $\mathbf{x}$ \\
\hline $\begin{array}{l}\text { Hazardous Waste } \\
(24 \mathrm{~h}, 8 \mathrm{~h} \text { OJT })\end{array}$ & $\mathbf{x}$ & $\mathrm{x}$ & $\sqrt{ }$ & $\mathbf{x}$ \\
\hline $\begin{array}{l}\text { Hazardous Waste Annual } \\
\text { Refresher }(8 \mathrm{~h})\end{array}$ & $\sqrt{ }$ & $\sqrt{ }$ & $\sqrt{ }$ & $x$ \\
\hline $\begin{array}{l}\text { Hazardous Waste Supervisors } \\
\text { Training }(8 \mathrm{~h})\end{array}$ & $\mathrm{x}$ & $\sqrt{ }$ & $\mathrm{x}$ & $\mathrm{x}$ \\
\hline $\begin{array}{l}\text { Hazard Communication } \\
\text { Training } \\
\text { (Contained in } 40 \text { or } 24 \mathrm{~h} \text { and } \\
\text { site-specific training) }\end{array}$ & $\sqrt{ }$ & $\sqrt{ }$ & $\sqrt{ }$ & $\mathbf{x}$ \\
\hline $\begin{array}{l}\text { Respiratory Protection } \\
\text { (required only if respirators } \\
\text { are worn; contained in } 40 \mathrm{~h} \text { ) }\end{array}$ & $\sqrt{ }$ & $\sqrt{ }$ & $\sqrt{ }$ & $\sqrt{ }$ \\
\hline $\begin{array}{l}\text { Respirator Issue } \\
\text { (required for respirator } \\
\text { issuers) }\end{array}$ & $\mathrm{x}$ & $\mathrm{x}$ & $\mathbf{x}$ & $\mathbf{x}$ \\
\hline $\begin{array}{l}\text { Waste Generation (required if } \\
\text { hazardous waste is generated) }\end{array}$ & $\sqrt{ }$ (at least one) & $\mathrm{x}$ & $\mathrm{x}$ & $\mathbf{x}$ \\
\hline Site Access Briefing & $\sqrt{ }$ & $\sqrt{ }$ & $\sqrt{ }$ & $\sqrt{ }$ \\
\hline $\begin{array}{l}\text { Routine Safety Briefings (at } \\
\text { least weekly) }\end{array}$ & $\sqrt{ }$ & $\sqrt{ }$ & $\mathrm{x}$ & $\mathbf{x}$ \\
\hline
\end{tabular}

$$
\begin{array}{lll}
\sqrt{ } & = & \text { Required } \\
\mathbf{x} & = & \text { Not required } \\
\text { OJT } & = & \text { on the job training }
\end{array}
$$

The 8-h Annual Hazardous Waste Refresher course is required to maintain currency in the 40-h and 24-h courses. It must be repeated annually by anyone who is required to have either of these courses. This course is mandated by the Hazardous Waste Operations and Emergency Response Standard (29 CFR 1910.120).

The Hazardous Waste Supervisor training is required for all personnel who directly supervise hands-on workers and for personnel who serve as SHSOs. This is an 8-h course that must be taken once. No refresher is required. The Superfund Amendments and Reauthorization Act (SARA)/OSHA 40-h course is a prerequisite.

Hazard Communication training is required for all site workers. This training must communicate the risks and protective measures for chemicals that employees may encounter. This requirement is met by taking GET, 40 or 24-h training, and the appropriate access briefing. This training must be refreshed as needed to remain current with the chemical hazards present on the job site. 
Waste Generation training is required for personnel who are responsible for the generation and satellite storage of hazardous waste. This training addresses the legal and operational requirements for the proper storage and labeling of hazardous waste.

Respiratory Protection Training is required for all individuals who wear respirators. It includes the basic procedures for proper respirator use. This training must be refreshed on an annual basis. Respirator users must also have medical clearance to wear respirators and must have passed a quantitative respirator fit test with the size and type of respirator to be used.

Respirator Issue training is required for any individual who issues respiratory protective devices. This training includes the information in Respiratory Protection Training as well as the requirements for proper storage, issuance criteria, and use limitations.

All personnel working on the project must have attended the pre-entry H\&S briefing. The briefing will cover site-specific hazards likely to be encountered and will include site-specific hazard communication instruction. Documentation of the topics covered and signatures of those attending will be entered in field logbooks. The site access H\&S Briefing will include the following:

- names of site H\&S personnel,

- site-specific hazard communication including location of MSDSs,

- HASP requirements,

- project-specific hazard controls,

- requirements of any relevant RWPs,

- emergency (contingency) procedures, and

- confined space recognition.

Site-specific routine safety briefings will be held at least weekly. These briefings will address changes in site conditions, additional hazard controls, or will be used to emphasize existing controls.

The training listed above is specific to the H\&S training requirements for this project and is not intended as a substitute for task-specific training. All project personnel who may be required to perform on-site monitoring for chemical and radiological contaminants shall be appropriately trained in the operation, calibration, and/or field check procedures for the applicable instruments. All project personnel shall have received appropriate on-the-job training for specific field activities and tasks they may be asked to perform.

\section{PERSONAL PROTECTIVE EQUIPMENT}

All PPE for site activities, tasks, and work zones is based on potential site-specific contaminants. Protective equipment may be selected for chemical and/or radiological hazards. In cases where both types of hazards are present, a combination of the two types of protective equipment will be selected so that adequate protection is provided for both types of hazards. Saranex-coated Tyvek@ or other similar materials are acceptable substitutes for cloth anti-C 
coveralls, chemical-resistant disposable gloves are acceptable substitutes for anti-C gloves, and nitrile or butyl rubber boots are acceptable substitutes for anti-C shoe covers (Totes()). This section emphasizes the programmatic requirements for PPE.

The level of protection selected for a particular task is based on the following:

- potential for exposure because of work being done;

- route of exposure;

- measured or anticipated concentration in the medium of concern; and

- toxicity, reactivity, or other measure of adverse effect.

In situations where the type of chemical, concentration, and possibilities of contact are not known, the appropriate level of protection must be selected based on the professional experience and judgment of H\&S personnel until the hazards can be further evaluated.

\subsection{UPGRADE OR DOWNGRADE OF PPE}

The SHSO may raise or lower the level of PPE worn by field personnel, depending on the site-specific hazards encountered in the field. The EMEF H\&S Manager and the subcontractor H\&S Manager must approve downgrades to PPE. If it is necessary to raise the level of PPE, or to evacuate the work site, the EMEF H\&S Manager and subcontractor H\&S Manager will be notified immediately. Criteria indicating a possible need for reassessment of PPE selection include the following:

- temperature extremes posing a serious hazard to employees wearing the required PPE,

- new hazards encountered,

- airborne concentration of chemical/radiological hazards changes, or

- work scope or work area changes.

\subsection{SITE PPE LEVELS}

Levels of protection that may be used to protect against chemical hazards are detailed below. See Table 2 for PPE requirements for specific tasks.

- Level D+ protective equipment

- Tyvek $\otimes$ clothing or Energy Systems-issued equivalent

- chemical-resistant (nitrile) disposable gloves

- disposable latex gloves

- safety shoes

- safety glasses with side shields

- Level D protective equipment

- coveralls/field clothes

- disposable latex (or nitrile) gloves for handling potentially contaminated material 
- safety shoes

- safety glasses with side shields

- Level D radiological contamination protective equipment

- anti-C coveralls

- safety shoes

- safety glasses (with side shields)

- skull cap or hood

- shoe scuffs

- disposable latex gloves

- nitrile disposable gloves

\section{MEDICAL SURVEILLANCE}

Employees working on hazardous waste-related investigations may be exposed to hazardous chemicals, various forms of radiation, noise hazards, biological hazards, and safety hazards. Personnel performing on-site, hands-on work will be medically qualified to perform the work and have medical approval to wear respirators, if required. A medical surveillance program is both essential and required per 29 CFR 1910.120(f) to assess and monitor workers' health and fitness for employment in this field.

\subsection{EMPLOYEES COVERED}

Medical clearance for hazardous waste work and enrollment in a medical surveillance program are required for project personnel who

- enter exclusion zones, contamination reduction zones, or radiological control areas;

- participate in sampling tasks, participate in sample preparation, perform equipment decontamination; or

- perform any work or enter any area where they could be exposed to hazardous waste.

\subsection{FREQUENCY OF EXAM}

The frequency of employee medical exams shall be as follows:

- prior to assignment;

- once every 12 months for each employee covered unless the attending physician believes a shorter or longer interval (not to exceed 2 years) is appropriate; 
- at termination of employment or reassignment to an area where the employee would not be covered;

- as soon as possible upon notification by an employee that (1) the employee has developed signs or symptoms indicating possible overexposure to hazardous substances or health hazards or (2) the employee has been injured or exposed above the PEL or published exposure levels in an emergency situation; and

- at frequencies required by site procedures, such as urinalysis before and after a project.

\subsection{MEDICAL EXAM CONTENT}

Medical examinations shall include a medical and work history (or updated history if one is available in the employee's file) with special emphasis on symptoms related to the handling of hazardous substances and health hazards and to fitness for duty, including the ability to wear any required PPE under conditions that may be expected at the work site.

The employee shall have access to the results of the medical examination and to any necessary explanations of the tests and findings.

\subsection{MEDICAL SURVEILLANCE FOR RESPIRATOR USERS}

Both 29 CFR 1910.134 and the American National Standards Institute (ANSI) standard Z88.2 have specific medical surveillance requirements independent of 1910.120. These requirements include an annual medical examination for respirator users. All site personnel required to wear respiratory protection will have met these medical examination and surveillance requirements.

\subsection{MEDICAL SUPPORT}

The Y-12 Plant Fire Department operates an ambulance service staffed by emergency medical personnel $24 \mathrm{~h}$ a day. Doctors and nurses are available during the day shift at the medical facilities. Injured personnel will be transported to the Oak Ridge Methodist Medical Center by ambulance during evening work shifts.

\section{MONITORING AND SAMPLING}

The action levels for organic vapor measurements listed in Table 5 have been set conservatively to compensate for monitoring instrument limitations.

Both historical site data and current well monitoring data indicate that chemical and radiological exposure or radiological contamination of site personnel is not a concern within the scope of this project. Organic vapor measurements will be conducted if the SHSO determines 
Table 5. Site monitoring action limits

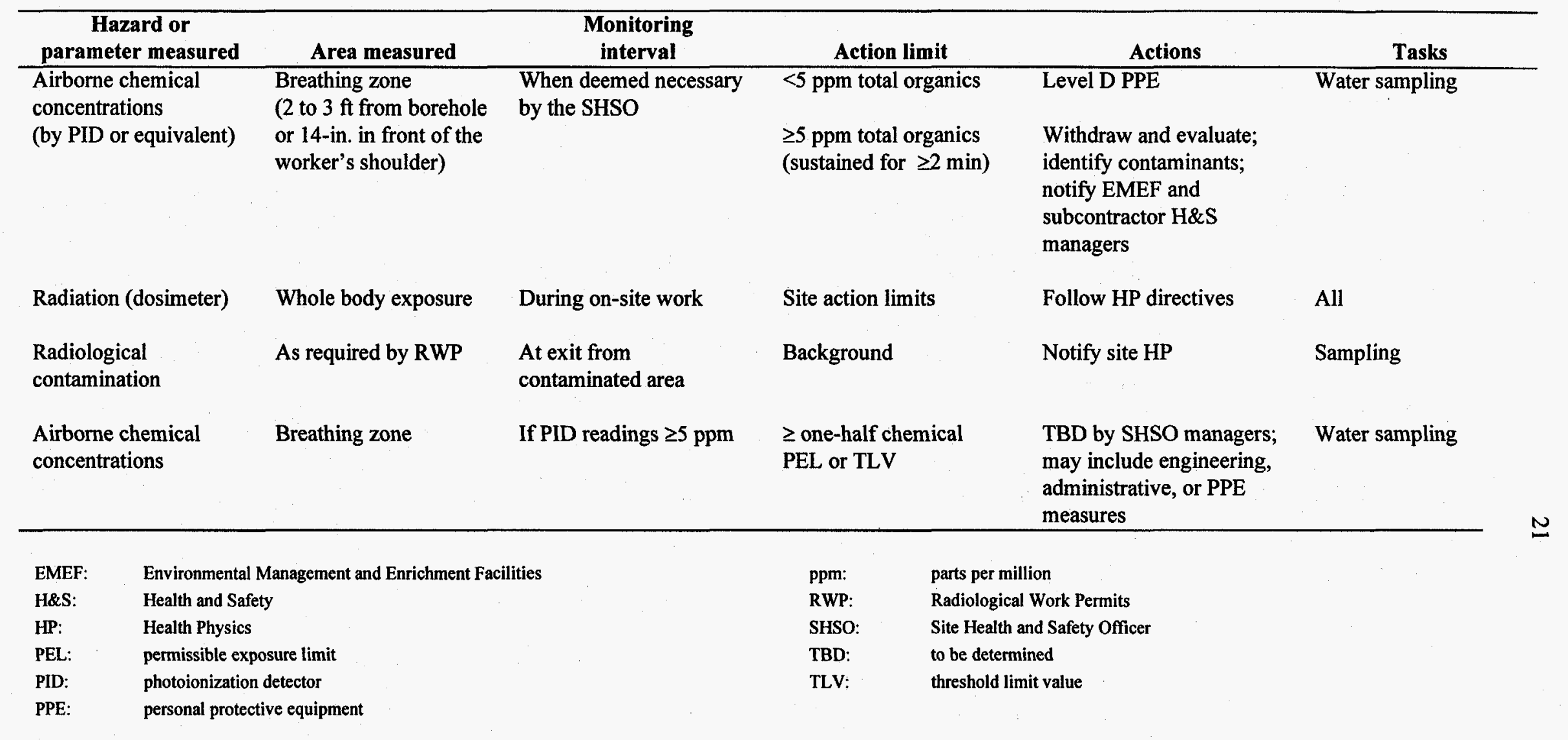


that an area or activity poses a potential for overexposure. The SHSO will make the determination based on the history of the sampling location, the presence of odors, and the existing ventilation. Although no radiological exposure is anticipated during planned site activities, an HP technician may periodically monitor site operations and site equipment for radiological contamination. If the HP technician determines the area requires radiological posting, work will cease until the area is appropriately posted or decontaminated. If required, the level of PPE will be upgraded by the SHSO and the HP technician.

The following types of routine monitoring will also be required:

- All personnel entering the site will participate in the Site Dosimetry Program. P-NAD or thermoluminescent dosimeters will be worn by personnel at all times while on site.

- Persons entering known or suspected contaminated areas will monitor or be monitored for contamination prior to exiting those areas.

- Upon notification by the EMEF Project Manager, the HP Department will perform pre-entry radiation scans of subcontractor vehicles and equipment that will enter contaminated areas.

- After notification by the EMEF Project Manager, an HP technician will perform radiation scans when the project vehicle(s) and equipment leave a contaminated area.

- Personnel entering contamination or high contamination areas will participate in the urinalysis bioassay program as directed by the site HP department.

- Personnel entering contamination or high contamination areas will participate in whole body counting or lung/chest counting as directed by the site HP department.

\section{SITE CONTROL MEASURES}

\subsection{PROJECT-SPECIFIC SITE CONTROL}

Site control will be appropriate to the anticipated site chemical, radiological, and physical hazards. Traffic cones will be placed around parked vehicles on roadways if field operations may obstruct the road. Work zones will be established by each field crew around the well(s), support vehicle(s), and the equipment decontamination area. Based on the minimal levels of contamination anticipated in this project, formal barricaded work zones will not be established unless monitoring data indicate the need for such barriers. Work crews and the SHSO will use signs (Hazardous Waste Operations or similar) and administrative controls to prevent the entry of untrained or unprotected personnel or visitors into the work zone. Site personnel will comply with the requirements of existing contamination areas.

All personnel entering the work zone will wear the prescribed level of protective clothing. Entry into the work zone is prohibited without the approval of the SHSO or unless the person desiring entry has attended a pre-entry H\&S briefing. The number of people and equipment in the work zone will be minimized to control physical hazards and the potential spread of 
contamination. All non-work activities (e.g., rest breaks) will take place in the support zone. Project visitors will be instructed to stay outside the work zone unless entry is approved by the SHSO.

\subsection{PERMITS AND PASSES}

To conduct this project, a number of permits and passes (such as for vehicles and communications) must be obtained and maintained. The EMEF Project Manager will be responsible for ensuring that all applicable permits are obtained before field activities begin.

\section{DECONTAMINATION PROCEDURES}

\subsection{PERSONNEL DECONTAMINATION}

Personnel decontamination procedures are designed to limit the contaminated materials workers may encounter and to limit the spread of contaminated materials from the work zone. Level $\mathrm{D}$ protection is normally used when the potential for personnel contamination is very low, as is the case with this project; therefore, formal decontamination of personnel will be conducted only if contamination is detected. As a minimum, personnel who have entered potentially contaminated areas will wash their hands prior to eating or drinking.

Should radioactive contamination be detected on an individual, the SHSO or the individual contaminated will contact HP immediately. HP personnel will supervise and assist in decontaminating the individual.

\subsection{EQUIPMENT DECONTAMINATION}

Decontamination of tools will be performed after each sampling. The equipment will be decontaminated as specified in the Sampling and Analysis Plan.

\subsection{DECONTAMINATION EQUIPMENT AND SUPPLIES}

Equipment that may be required for decontamination of the equipment is listed here.

- Moving-style utility truck(s)

- Traffic cones

- Heavy-duty plastic sheeting

- 30-gal trash cans

- Trash bags

- Paper towels

- Kimtowels

- Hand sprayers with Teflon® tip nozzles

- New DOT 17E drum for wastewater 
- Wooden pallets

- Binding straps for drums

- Liquinox

- Type II ASTM water

- Plastic brushes

- Plastic tub(s)

- Funnel

- Spill response kit

- Buckets

- Ground cover

- Tape

- Storage crates

- Garden hose

The tap water supply carboy may need to be filled (with a garden hose) daily. A separate drum funnel from the one used to transfer wastewater (as described in Sect. 9.2) will be used to transfer clean tap water for decontamination activities to the hand sprayers.

\section{SITE OPERATING PROCEDURES}

This section presents general safety rules that apply to the entire project. The provisions of this HASP are mandatory for all on-site employees and visitors.

- The SHSO will ensure that a copy of this HASP is on site and available to site personnel, upon request.

- All project personnel will be required to provide the SHSO with documentation of successful completion of all training and medical surveillance requirements.

- The SHSO and project personnel are responsible to suspend or stop work if unsafe conditions occur.

- Good housekeeping will be practiced.

- Field crew members must be familiar with the physical characteristics of investigation sites, including

- wind direction in relation to the individual's location;

- accessibility to associates, equipment, and vehicles;

- available means of communication;

- any areas of known or suspected contamination;

- site access requirements; and

_ the location of the nearest drinking water sources and sanitary facilities.

- All injuries will be reported to the SHSO and/or to the Field Operations Manager, no matter how minor. 
- Each person will make his/her presence known to the SHSO or Field Operations Manager daily, and personnel changes will be noted in the field logbook. A list of workers on the job site will be maintained in the field logbook for accountability.

- Copies of MSDSs for all hazardous chemicals brought on site will be maintained in the work area. MSDSs will be available to all employees for review during each work shift.

- Contact with potentially contaminated substances will be avoided. Personnel will avoid walking through puddles, pools, and mud; kneeling on the ground; and leaning or sitting on equipment or the ground.

- Spills will be prevented to the extent possible. If a spill occurs, the material will be contained with the on-site spill kit.

- Splashing of contaminated materials will be prevented or minimized.

- All containers received on site will be inspected to ensure the following:

- clear labeling as to the contents;

- the appropriate hazard warnings; and

- the name and address of the manufacturer.

- All secondary containers of hazardous chemicals will be labeled with either an extra copy of the original manufacturer's label or with generic labels that have a block for content identity and for the hazard warnings.

- A qualified alternate shall be designated if the SHSO is absent.

- A buddy system will be established that ensures that all field personnel will remain in visual contact with at least one other person on site so that they may be able to assist in the event of an emergency.

\subsection{SAFETY EQUIPMENT AND SITE COMMUNICATIONS}

The safety equipment that may be required during the scope of this project for personal protection, work zone safety, and contaminant monitoring is listed here.

- PPE

- safety glasses (with side shields)

- safety shoes

- work gloves

- field work clothes (reusable, washable)

- disposable chemical-resistant (nitrile) gloves

- disposal latex gloves 
- Work zone equipment

- traffic cones

- first aid pack

- cellular phone/radió

- assembly point locator map

- fire extinguisher

- hand soap or towelettes

- drinking water cooler

- spill kit appropriate to foreseeable spills

- eye wash station

The means of on-site and plant communication that will be available on site during this project will consist of two-way radios and/or a cellular telephone.

\section{CONTINGENCY PLAN}

The Y-12 Plant emergency response organization will be contacted for response to all emergencies at the project sites. The Plant or Laboratory Shift Supervisor will coordinate all emergency response operations. All personnel working on site will be trained to recognize and report emergencies to the SHSO. The SHSO will notify the emergency response organization for assistance.

\subsection{EMERGENCY PHONE NUMBERS}

A cellular phone will be present in the field and available for use when workers are not close to a plant phone or radio. Note that 911 will not access the Plant or Laboratory Shift Supervisor from a cellular phone.

PLANT OR LAB SHIFT SUPERVISOR

Plant Phone (Site telephone number)

911

Cellular Telephone Number

Y-12: 574-7172

\subsection{REPORTING AN EMERGENCY}

\subsubsection{Telephone}

- Describe the type of emergency.

- Identify the location of the emergency.

- Identify who is calling.

- Identify the number on the phone being used.

- Tell whether an ambulance is needed.

- Listen to and follow any instructions given.

- Do not hang up until after the Emergency Control Center has hung up. 


\subsubsection{Fire Alarm Pull Boxes}

Pulling a fire alarm box automatically transmits the location of the emergency to the site Fire Department and the Emergency Control Center. The person pulling the alarm should remain at the alarm box and supply any needed information to the responding emergency squad.

\subsection{ALARM SIGNALS}

Each field team will have a compressed gas horn that will be used to signal to other project personnel in the event of accidents or emergencies. Any use of the horn will indicate an immediate need for help.

\subsection{EVACUATION PROCEDURES}

Field crews will be familiar with the evacuation routes designated by the SHSO. In the event of an evacuation, personnel will proceed to the predetermined assembly point and wait for further instructions. Figure 1 shows the evacuation routes at Y-12 Plant. The field operations manager and crew will follow instructions given by the Emergency Response Team upon its arrival.

\subsection{EMERGENCY EQUIPMENT}

Several types of emergency equipment will be maintained at the work site, including the following items:

- first aid kit suitable to treat minor injuries such as cuts and abrasions;

- emergency communication equipment;

- pressurized shower/eye wash to meet ANSI standards; and

- basic spill kit suitable to handle small spills of decontamination fluids, hydraulic fluid, or fuels.

\section{REFERENCES}

NIOSH (National Institute for Occupational Safety and Health) 1994. Pocket Guide to Chemical Hazards, U.S. Department of Health and Human Services, Public Health Service, Centers for Disease Control, June.

ACGIH (American Conference of Governmental Industrial Hygienists) 1996. 1996 Threshold Limit Values for Chemical Substances and Physical Agents and Biological Exposure Indices, ISBN: 0-936712-99-6.

29 CFR 1904, Recording and Reporting Occupational Injuries and Illnesses 





29 CFR 1910, Safety and Health Regulations for General Industry

29 CFR 1926, Safety and Health Regulations for Construction 


\section{DISTRIBUTION}

1. L. V. Asplund

2. J. A. Davis

3. V. M. Forsberg

4. C. S. Haase

5. J. C. Henderson

6. R. L. Marcum

7. L. E. May

8. H. C. Newson

9. P. T. Owen

10. L. B. Raulston

11. L. G. Schaffer -

12-14. J. H. Vanderlan

15. D. B. Watson

16. S. A. White

17. M. C. Wiest

18. File-EMEF DMC-RC

19. R. Adkisson, Entech, 560 Oak Ridge Turnpike, Suite 2, Oak Ridge, Tennessee 37830

20. M. Leavitt, Science Applications International Corporation, 800 Oak Ridge Turnpike, P.O. Box 2502, Oak Ridge, Tennessee 37831

21. D. Moss, Science Applications International Corporation, 800 Oak Ridge Turnpike, P.O. Box 2502, Oak Ridge, Tennessee 37831

22. B. Sams, Jacobs Engineering Group, Inc., 125 Broadway Avenue, Oak Ridge, Tennessee 37830 